\title{
Nutritional Recommendations for People with Type 1 Diabetes Mellitus
}

\author{
Authors \\ Diana Rubin 1, 2, Anja Bosy-Westphal ${ }^{3}$, Stefan Kabisch", Peter Kronsbein ${ }^{5}$, Marie-Christine Simon ${ }^{6}$, Astrid Tombek ${ }^{7}$, \\ Katharina S. Weber ${ }^{8}$, Thomas Skurk ${ }^{9}$, for the Nutrition Committee of DDG
}

\author{
Affiliations \\ 1 Vivantes Hospital Spandau, Berlin, Germany \\ 2 Vivantes Humboldt Hospital, Berlin, Germany \\ 3 Institute of Human Nutrition, Faculty of Agriculture and \\ Nutritional Sciences, Christian-Albrechts University of \\ Kiel, Kiel, Germany \\ 4 German Institute of Human Nutrition Potsdam-Re- \\ hbrücke, Potsdam, Germany \\ 5 Faculty of Nutrition and Food Sciences, Niederrhein University \\ of Applied Sciences, Campus Mönchengladbach, Germany \\ 6 Institute of Nutrition and Food Sciences, Rheinische \\ Friedrich-Wilhelms-University Bonn, Bonn, Germany \\ 7 Diabetes Clinic Bad Mergentheim, Germany \\ 8 Institute for Epidemiology, Christian-Albrechts University \\ of Kiel, Kiel, Germany \\ 9 ZIEL - Institute for Food \& Health, Technical University \\ Munich, Munich, Germany
}

published online $\quad 29.12 .2020$

\section{Bibliography}

Exp Clin Endocrinol Diabetes 2021; 129: S27-S43

DOI 10.1055/a-1284-6036

ISSN 0947-7349

(C) 2020. Thieme. All rights reserved.

Georg Thieme Verlag KG, Rüdigerstraße 14,

70469 Stuttgart, Germany
German Diabetes Association: Clinical Practice Guidelines This is a translation of the DDG clinical practice guideline published in Diabetologie 2020; 15 (Suppl 1): S120-S138. DOI: 10.1055/a-1245-5623.

\section{Correspondence}

Prof. Dr. Diana Rubin

Zentrum für Ernährungsmedizin

Vivantes Klinikum Spandau

Neue Bergstraße 6

13585 Berlin

und

Vivantes Humboldt Klinikum

Am Nordgraben 2

13509 Berlin

Germany

Diana.Rubin@vivantes.de

$\begin{array}{ll}\text { AB BREVIATIONS } \\ \text { ADI } & \text { Acceptable Daily Intake } \\ \text { BMI } & \text { Body mass index } \\ \text { CU } & \text { Carbohydrate unit } \\ \text { CGM } & \text { Continuous glucose monitoring } \\ \text { CHO } & \text { Carbohydrate } \\ \text { DASH } & \text { Dietary Approaches to Stop Hypertension } \\ \text { DDG } & \text { Deutsche Diabetes Gesellschaft (German } \\ & \text { Diabetes Association) } \\ \text { DGE } & \text { Deutsche Gesellschaft für Ernährung (German } \\ & \text { Nutrition Society) } \\ \text { EFSA } & \text { European Food Safety Authority } \\ \text { EI } & \text { Energy intake } \\ \text { FPU } & \text { Fat protein unit }\end{array}$

$\begin{array}{ll}\text { GFR } & \text { Glomerular filtration rate } \\ \text { GI } & \text { Glycemic index } \\ \text { HDL } & \text { Higfh-density lipoproteins } \\ \text { HEI } & \text { Healthy Eating Index } \\ \text { HLA } & \text { Human leukocyte antigen } \\ \text { ICT } & \text { Intensified conventional insulin therapy } \\ \text { iscCGM } & \text { Intermittent-scanning CGM } \\ \text { LDL } & \text { Low-density lipoproteins } \\ \text { LPS } & \text { Lipopolysaccharide } \\ \text { MNA } & \text { Mini Nutritional Assessment } \\ \text { MUFA } & \text { Monounsaturated fatty acid } \\ \text { n-9RCFA } & \text { Red cell phospholipid fatty acids } \\ \text { PUFA } & \text { Polyunsaturated fatty acid } \\ \text { RCFA } & \text { Red cell phospholipid fatty acid } \\ \text { RCT } & \text { Randomized,. controlled trial }\end{array}$

GFR

$$
\text { GI }
$$

HDL

$$
\text { HEI }
$$$$
\text { HLA }
$$

\section{iscCGM}$$
\text { MNA }
$$

MUFA n-9RCFA

RCFA 


$\begin{array}{ll}\text { rtCGM } & \text { Real-time CGM } \\ \text { SFA } & \text { Saturated fatty acid } \\ \text { SF-MNA } & \text { Short form Mini Nutritional Assessment } \\ \text { T1Dm } & \text { Type } 1 \text { diabetes mellitus } \\ \text { T2Dm } & \text { Type } 2 \text { diabetes mellitus } \\ \text { VLCD } & \text { Very low calorie diet } \\ \text { WHO } & \text { World Health Organization }\end{array}$

\section{Preamble}

This practice recommendation is aimed at all people with type 1 diabetes and all professional groups that care for people with type 1 diabetes.

The most recent "Evidence-based dietary recommendations for the treatment (and prevention) of diabetes mellitus" are from the year 2005 [1]. Since then, the aspect "nutrition and diabetes" has been covered by the DDG as a sub-chapter within the framework of numerous superordinate guidelines and practice recommendations.

In accordance with the demand for individualization of therapy, counseling, empowerment and diabetes self-management [2,3] and in view of the consensus report "Nutrition Therapy for Adults with Diabetes or Prediabetes" [4, 5], the Nutrition Committee of the DDG has set itself the goal (planned publication 2021) of presenting evidence-based practice recommendations on nutrition for the various (sub)types of diabetes mellitus in a summarizing body of work. A separate presentation according to diabetes types and forms of treatment is considered necessary, as the therapeutic significance of nutrition differs significantly in each case and must be considered against the background of different drug therapy components.

This paper presents the work done so far on selected nutritional aspects in the treatment of type 1 diabetes.

Nutritional recommendations should consider the individual life and nutritional habits of each person with diabetes mellitus with the goal of maintaining the nutritional regime for as long as possible.

Many variables, such as exercise, stress, meal composition, etc., have an impact on blood glucose values with a high individual variability.

Recommendations to modify the regular diet - and thus limit it from the patient's perspective - are justified if this lifestyle change can be used to achieve a clinically-relevant health benefit or to prevent a health threat based on evidence.

It can be observed that authors of observational and experimental studies with a small number of test persons repeatedly derive general recommendations from their study results, although this is not justified, especially when it comes to recommendations for the preference of singular foods (groups). In this sense, the publication planned for 2021 should help to focus from the encyclopedia of current nutritional recommendations on those that are evidence-based and clinically/healthily relevant for the specific therapy.

In the practice recommendations on diet in type 1 diabetes presented here, it is clear that the evidence for general recommendations is limited. Individual testing (CGM) of the glycemic effects of food intake is important in many aspects of nutrition. The empirical values thus determined - in addition to the patient's own food preferences - provide a suitable basis for individual food selection and the appropriate strategy for insulin application.

\section{Nutritional Aspects in Relation to Glycemia and Glycemic Target Parameters}

\section{Estimation of carbohydrate quantities}

Recommendation

Patients should

- Correctly estimate the amount of $\mathrm{CHO}$ ingested per meal to an accuracy of 1 carbohydrate unit (CU), equivalent to $10 \mathrm{~g}$ of $\mathrm{CHO}$ (as a basis for algorithm-guided dosing of prandial insulin)

- Know their individual postprandial glucose trends.

- Perform repeated testing of a standardized meal ideally via a rtCGM or iscCGM

\section{Comment}

Evidence-based studies are hardly available, but practical experience shows the following results. It is of crucial importance for the treatment of type 1 diabetes that patients are enabled to assess the glucose efficacy of their diet in order to adjust insulin dosage accordingly [6-8]. Structured training should provide the basis for this. It should take place repeatedly $[6,8]$.

There is no recommendation on the amount of carbohydrates $[7,8]$. How much of each macronutrient should be eaten has to be considered on an individual basis. The evaluation of postprandial glucose trends via rtCGM and iscCGM can help to choose the right bolus variant or the correct injection-to-meal interval [9]. Especially in cases of gastroparesis, the control of different bolus variants can ensure an individually-adapted trend.

Patients with a fixed dose must estimate the correct amount of carbohydrate in their meals [7]. It is also important to estimate carbohydrate quantities correctly during pregnancy for moderate postprandial glucose trends.

Individualized nutrition and the corresponding adaptation of insulin dose algorithms can lead to a better acceptance of the disease. This is particularly important for children and adolescents.

\section{SCIENTIFIC BACKGROUND}

There is no hard evidence for assessing $\mathrm{CHO}$. In a pilot study, 256 people with T1Dm were divided into two groups: trained and untrained in nutrition. When comparing the two groups, patients with training had significantly lower HbA1C $(7.8+/ - \pm 1.3-7.4+/ - \pm 0.9 \%$ vs. $7.5+/ - \pm 0.8-7.5+/ \pm 1.1)$ and less hypoglycemia [10]. In a study of 43 children aged 8.5-17.7 years with ICT, postprandial glucose trends were examined as a function of carbohydrate levels. It was shown that it is necessary to accurately estimate to within $10 \mathrm{~g}$ of carbohydrates to remain at the postprandial target [11]. Another study with type 1 patients exhibited similar results and the importance of good training for carbohydrate calculation [12]. The importance of this was also demonstrated in a study of 102 children and adolescents aged 8.3-18.1 years. Here the mean gram error was negatively significantly correlated with the HbA1c $(r=-0.7)$. It was also shown that the longer the children calculated carbohydrates, the greater the mean 
percentage error $(r=0.173)$. The authors therefore concluded that children and their caregivers can estimate well, but that a gram calculation is not better than a portion calculation. Repeated age-related training is necessary to reduce the error rate [13]. It has also been shown that for a good postprandial setting, accurate carbohydrate calculation within $10 \mathrm{~g}$ is important. In this study of 30 children (8-18 years) with CSII therapy, it was also shown that accuracy is not related to quantity.

\section{Glycemic index}

\section{Recommendation}

- The individual testing of the glycemic response (by glucose self-monitoring) to the preferred foods and meals is of great importance in order to develop and implement an adequate individual prandial insulin application strategy (including injection timing and application mode for CSII).

- There is no evidence that generally giving preference to low $\mathrm{Gl}$ carbohydrate carriers helps people with T1Dm achieve their therapeutic goals.

- For individual meals, it is recommended to cover carbohydraterich foods with a low GI (below approx. 30) with significantly less prandial insulin in order to prevent hypoglycemia, especially in the case of an almost normoglycemic metabolic control.

\section{Comment}

There are many GI tables in circulation, but only a few published in scientific papers [14-16] are suitable to be used to define the Gl of carbohydrate-containing foods. In order to assess the GI, it should be taken into account that the $\mathrm{Gl}$ values are basically average values from several studies. Variety, degree of ripeness, place of cultivation/production and, if applicable, recipe of a foodstuff all have a decisive influence on the $\mathrm{Gl}$. For bananas, for example, an average Gl of 48 (ref. glucose $=100 \%$; volunteers: type 2 diabetes) is reported - with a range of GI 30 to Gl 58 for the 9 studies considered [14]. For cooked white rice (mean GI: 59), the range even goes from GI 43 to GI 112 [14].

Studies in adults with type $\mathbf{1}$ diabetes under experimental conditions that compared the blood glucose response to (single) whole meals with low vs. high GI [17-19] have found significantly lower blood glucose responses after low GI meals and observed hypoglycemia when low GI carbohydrate carriers (lentils) are covered with the usual dose of mealtime insulin [17]. A study of blood glucose responses over a full day (4 meals per day) with insulin pump therapy [20] has shown that near-normoglycemic metabolic control is achieved regardless of $\mathrm{Gl}$ when patients adequately adjust their insulin administration.

Medium- and long-term studies on the effects of a low-Gl diet vs. a high-Gl diet in adults with type 1 diabetes under everyday conditions are scarce; they date back mainly to the 1990s and have recruited few subjects $[18,21]$.

In a meta-analysis from 2010 [22], 2 studies in adults with type 1 diabetes were included; however, they have methodological deficiencies and are not relevant for statements on $\mathrm{Gl}[23,24]$. A recent review of the relevance of the $2018 \mathrm{Gl}$ in adults did not include people with type 1 diabetes [25].
Studies in children and adolescents with type 1 diabetes were mainly conducted on small numbers of subjects and under experimental conditions [26-28], so that no fundamental benefits and no favorable recommendations can be derived from them. A study from the year 2001 [29] examined 2 different dietary recommendations for 52 weeks in children with type 1 diabetes. The results of this study are often used as an argument for the benefit of a low GI diet. However, this is not justified, since the study did not intend to compare a low-GI diet with a high-GI diet, but primarily compared a diet with a fixed carbohydrate distribution ("carbohydrate framework") vs. a diet with flexible carbohydrate intake. The evaluation of the subjects' dietary protocols showed identical values for the mean Gl of the consumed carbohydrate carriers for both groups.

Overall, there is no evidence from the available multi-week dietary studies that people with type 1 diabetes should give preference to low Gl carbohydrate carriers in their diet.

With regard to the consumption of individual foods/meals (according to the "acute food/meal studies" and clinical experience), special consideration of foods containing carbohydrate with a very low GI (below approx. 30) or a very high $\mathrm{Gl}$ (above approx. 90) seems advisable.

Foods with a very low GI (below approx. 30: e. g. pulses, nuts) should be covered with less prandial insulin to avoid hypoglycemia in the case of almost normoglycemic preprandial blood glucose values, while those with a very high GI (especially sugar-sweetened drinks) are more likely to be reserved for self-treatment of hypoglycemia. Individual testing of the glycemic response (through glucose self-monitoring) to the preferred foods and meals is of great importance, similar to finding an optimal personal strategy for physical activity in diabetes.

In this way it is possible to determine the factors, in addition to the amount of carbohydrates ingested, which affect the postprandial blood glucose response, and these include, in addition to the $\mathrm{Gl}, \mathrm{e.g}$. fluid intake, fat, protein, alcohol content of accompanying foods, preprandial glycemia, general glycemic control, body weight, physical activity, absorption of insulin from the application site.

The evaluation of postprandial glucose trends via rtCGM and iscCGM can help to select the appropriate insulin dose and bolus variant or injection-to-meal interval. People with ICT therapy can act with the options of injection-to-meal intervals or split boluses. People with CSII therapy can act via dual bolus/multiwave boluses or delayed boluses and injection-to-meal intervals. Basic criteria for the decision are initial glucose levels, carbohydrate amounts (CHO) and the $\mathrm{Gl}$ of the diet. (Nocturnal) hypoglycemic events must be prevented especially resulting from evening meals with a very low Gl.

The blood glucose response is always seen in combination with the insulin strategy. In practice, it has proven to be useful to vary the time of injection or, in the case of CSII, the insulin application mode for certain foods and starting situations according to personal experience.

\section{Insulin requirements for protein and fat-rich meals}

\section{Recommendation}

- There is no evidence that people with type 1 diabetes generally estimate their meals quantitatively for energy, fat and protein content in order to derive an additional insulin bolus.

- The blood glucose response to very high-fat or high-protein meals varies from person to person and should be determined 
and documented by self-monitoring. On this basis, an additional insulin requirement may be developed individually.

- Increasing the prandial insulin dose for high-protein evening meals increases the risk of night-time hypoglycemia.

- People with type 1 diabetes should be able to assess the effect of fats and proteins on glucose levels.

\section{Comment}

Protein-rich meals also result in a certain insulin requirement and can lead to postprandial delayed and sustained hyperglycemia [3032]. With a carbohydrate intake of about 50 energy percent and the remaining distribution to fat and protein, about $50 \%$ of the total insulin is distributed to the fast-acting mealtime insulin and $50 \%$ is covered by the basal supply. People with type 1 diabetes who have a low carb diet have a correspondingly higher basal rate and a lower proportion of mealtime insulin. However, there are situations in which low carbohydrates, high fat and protein are eaten with the main meal or in which a high proportion of fat and protein is taken in the evening via nuts, for example. In these cases, a simple bolus via mealtime insulin is usually not sufficient. One way to cover the slow glucose increase as a result of such meals would be, for example, a correction with fast-acting mealtime insulin at a later time. With CSII therapy, it would also be possible to give a delayed bolus. In order to know how much bolus to give, the FPU (fat-protein unit) was propagated as a measure.

According to Pankowska, $100 \mathrm{kcal}$ of fat and protein are calculated as one CU and, depending on the amount of FPU, are delivered as a delayed bolus for a maximum of $8 \mathrm{~h}[30,33]$. In practice and in various studies, however, it has been observed that this formula cannot be used equally well for all people with type 1 diabetes. In a paper by Hermann, a calculation with $200 \mathrm{kcal}$ corresponding to one CU was compared [34]. In this work it was confirmed that hardly any formula can be applied equally well to all people with type 1 diabetes. In a pilot study with nuts, the same result was also obtained [35]. Glucose increases can be observed after eating foods rich in fat and protein, but the insulin dose must be determined and trained individually.

\section{Fibers and glycemic control}

Recommendation

- There is not yet sufficient evidence that people with type 1 diabetes should eat a high-fiber diet to achieve their glycemic therapy goal.

- Possible effects of a fiber-rich diet on other health-promoting aspects (e. g. cardiovascular diseases, intestinal health, weight management) should be considered separately and individually.

- The increased consumption of high-fiber foods seems to have a positive effect on the trend of postprandial glycemia, but the recommendations for fiber intake are based on those for the general population (30 g per day).

\section{Comment}

A fiber-rich diet is generally considered to be beneficial to health, especially in the case of diabetes mellitus. The German Nutrition Society (Deutsche Gesellschaft für Ernährung) recommends a daily intake of at least $30 \mathrm{~g}$ of dietary fiber for the general population, which is not achieved by the average German population [36]. So far, there are no evidence-based recommendations for a certain amount of dietary fiber in diabetes. Although many studies have been designed for type 2 diabetes, there is often no explicit differentiation between diabetes types, although there are certain aspects that require separate consideration [37].

In later life, for example, the study situation for high-fiber diets (DASH diet, vegetarian, vegan) is inconsistent regarding, e. g., renal endpoints, but there is a small indication to the benefits of vegetarian diets in a systematic review [38]. Lower blood pressure [39] and a lower inflammatory load are positive outcomes of higher dietary fiber intake in T1Dm [40].

In terms of weight management, dietary fiber has a firm position, at least in type 2 diabetes, when consumed as part of a diet that has a low glycemic index [41]. Fibers contribute to a reduction in energy density and a lowering of the glycemic index and positively modulates the blood glucose profile. Observational studies in subjects with T1Dm have also shown an association with lower HbA1c levels, but the association was only mildly significant, possibly explained by the suboptimal amount of dietary fiber ingested (mean value $16 \mathrm{~g}$ fiber per day) [42].

To investigate the influence of the microbiota, prebiotics were used to improve bacterial diversity. Inulin led to an increase in bifidobacteria in young type 1 diabetes patients ( $<17$ years) and simultaneously to a higher C-peptide value than in the control group [43].

\section{Supply of sucrose and fructose}

\section{Recommendation}

- Beyond general recommendations, there is no evidence of specific recommendations for reducing added sucrose in individuals with T1Dm.

- Foods containing naturally occurring sugars (glucose and fructose) should not be restricted in the diet of people with type 1 diabetes.

- The evidence base for the recommendation of a reduction of added fructose is uncertain.

\section{Comment}

The evidence for adult individuals with T1Dm regarding recommendations for sucrose and fructose intake is limited. With regard to the intake of added sugar, current evidence-based guidelines generally recommend that people with diabetes mellitus minimize their intake of added sugar and replace it with foods with a higher nutrient density [4, 6, 7].

The majority of the European professional societies express the recommendation to reduce the supply of sugar (primarily free or added sugars) for the general population as well. Recommendations that state a suggested upper limit usually set this at $10 \mathrm{El} \%$ for adults and $5 \mathrm{El} \%$ for children $>2$ years [44]. However, according to the dietary recommendations of the American Dietetic Association for people with T1Dm and T2Dm, sucrose intake of 10-35 El\% has no negative effects on glycemic or lipid response when starch isocalorically replaces sucrose [45]. A similar conclusion, namely that a sugar intake at a level common in human nutrition does not lead to undesirable metabolic health effects, is drawn by Rippe et al. in their review of randomized controlled trials [46].

In a randomized, controlled trial in which 33 persons with T1Dm received either a saccharose-free or a saccharose-enriched diet (mean sucrose or fructose intake after the intervention: 2 vs. 27 
El\% or 6 vs. $9 \mathrm{El} \%$ ) over 3 months, it was also confirmed that although higher concentrations of inflammatory markers (C-reactive protein) were detectable in subjects with higher sucrose intake, the two groups did not differ in insulin requirements, glycemic control, anthropometric parameters, body composition, or triglyceride concentrations [47].

\section{SCIENTIFIC BACKGROUND}

The intake of fructose with regard to diabetes mellitus is intensively discussed due to the metabolism deviating from glucose. Since fructose is first metabolized in the liver, it does not lead to an increase in blood glucose levels, unlike glucose, and thus does not trigger the release of insulin. Furthermore, fructose is converted to free fatty acids during de novo lipogenesis, but only to a small proportion of about 1-5\% [48]. Systematic reviews and meta-analyses, which included individuals with T1Dm, as well as a randomized controlled trial with T1Dm patients, show lower postprandial peaks for blood glucose, lower total cholesterol concentrations, no differences in other serum lipid parameters, but possibly increased uric acid concentrations and oxidative stress in an isocaloric comparison of fructose with other carbohydrates [49-52]. Due to the hepatic metabolism of fructose, its influence on hepatic insulin sensitivity is also widely discussed. The evidence for persons with and without T2Dm shows that only a very large increase in fructose intake to approximately $80 \mathrm{~g} / \mathrm{d}$ leads to a moderate reduction in hepatic insulin sensitivity, without an accompanying clinically-significant increase in fasting blood glucose concentrations [53].

In summary, the rationale for a reduction in sucrose and fructose intake for adults with T1Dm due to a lack of evidence for this group of people is primarily derived from the evidence for the general population or, more generally, people with diabetes and is based on the recommendations of a balanced diet: although an isocaloric replacement of foods containing sucrose or fructose with other carbohydrates may have similar effects on blood glucose levels or other metabolic parameters, consumption should be minimized in favor of foods with a higher nutrient density [54] and the selected foods containing carbohydrates in the diet should ideally be rich in fiber, vitamins, minerals and low in added sugars, fats and sodium [55]. For children and adolescents with T1Dm, the following study results can be added to justify a reduction in sucrose and fructose intake: data from a review and observational study show that a higher sugar intake is associated with the development of T1Dm in childhood [56] and that the late phase of disease development in particular could be accelerated [57]. In adolescents with manifest T1Dm, fructose intake was directly associated with triglyceride concentrations [58]. However, the intake of naturally-occurring sugars (within the normal range of the diet) was associated with improved parameters of glycemic control, and Nansel et al. conclude that foods containing this source of sugar do not have negative effects on blood glucose control in adolescents with T1Dm [59].

\section{Use of sweeteners}

\section{Recommendation}

- Sweeteners can be useful as an occasional addition to foods and beverages as part of a diabetes-compliant diet and insulin therapy and are harmless to health as long as they are consumed below the respective maximum amounts.

\section{Comment}

The topic of "sweeteners" is a recurring topic of discussion in nutrition. Sweeteners are synthetically produced or natural compounds with a high sweetening intensity, which are metabolized independently of insulin and are not cariogenic. Even though the calorie content of some sweeteners is $4 \mathrm{kcal} / \mathrm{g}$, just like conventional sugar (sucrose), they are only used in very small amounts in the milligram range due to their high sweetening power, so that calorie intake is negligible. As additives, sweeteners are subject to a health assessment by the European Food Safety Authority (EFSA) prior to approval, which determines acceptable daily intakes (ADI). The ADI value indicates the amount of an additive that can be ingested daily per kilogram of body weight over a lifetime without causing health risks.

Even after approval, the EFSA continues to further investigate the sweeteners if necessary. Although sweeteners are therefore considered to be harmless to health according to the current state of knowledge, possible long-term health consequences in particular have been discussed repeatedly for many years.

The reservation of an increased cancer risk from sweeteners is mainly based on older studies, which are based on results from animal experiments. However, these studies used very high amounts of the sweetener in question, far in excess of the acceptable daily intake for humans, so the results are not transferable to humans. According to recent animal studies and available human data, there is no evidence of an increased cancer risk from sweeteners if the ADI values are adhered to $[60,61]$.

An increase in weight caused by sweeteners has not yet been scientifically proven. The clinical studies on this topic sometimes diverge considerably. Epidemiological studies predominantly conclude that sweetener consumption is associated with an increased risk of excess weight or obesity. By contrast, studies with higher evidence levels tend to indicate a weight-neutral effect or that sweeteners are a tool that can be used with limitations for reducing energy intake and weight control $[62,63]$.

Some observational studies show a positive association between sweetener consumption and the risk of type 2 diabetes and other cardiometabolic diseases, but the bias of excess weight and reverse causality is often a problem $[61,63,64]$.

Regarding the effect of sweeteners on appetite, intervention studies show that sweetened beverages have an effect similar to water on appetite and energy intake $[63,65,66]$. A recent review of various studies investigating the effect of sweeteners on the appetite and consumption of sweetened products also showed no increase in either appetite or consumption [67].

With regard to the effects on human intestinal microbiota, the data are limited and do not provide sufficient evidence that sweeteners at the levels relevant for human consumption have a negative impact on the microbiota $[63,68]$. 
The potential benefit of sweeteners in patients with type 1 diabetes is mainly due to their use as a substitute for sugar without triggering a glycemic response. Despite controversial discussions, current research provides collective evidence that the consumption of sweeteners has no adverse effects on blood glucose and insulin regulation (HbA1c, fasting and postprandial glucose and insulin levels) in people with and without diabetes [63,69].

In general, research on sweeteners is hampered by the chemical and metabolic heterogeneity of sweeteners, their-at timescombined use in different product groups and the changing trends in consumer behavior $[61,70]$.

\section{Effective blood glucose food for hypoglycemia}

\section{Recommendation}

- Persons with T1Dm and mild hypoglycemia (can be treated by the patient) should consume 15-20 g of rapidly absorbable carbohydrates. This measure should be repeated after $15 \mathrm{~min}$ if blood glucose concentrations remain low.

- Persons with T1Dm and severe hypoglycemia experiencing confusion or clouding of consciousness should consume $30 \mathrm{~g}$ of rapidly absorbable carbohydrates. In individual cases, it may be necessary to supplement with $15-20 \mathrm{~g}$ of slowly resorbable carbohydrates.

\section{Comment}

Rapid carbohydrates such as oral glucose in solid and liquid form are particularly suitable for treating hypoglycemia. Furthermore, saccharose-containing beverages such as lemonades and juices can be used.

In some cases, slowly resorbable carbohydrates can contribute to the stabilization of blood glucose depending on the situation [6-8].

In order to avoid hypoglycemia in advance, in special situations, quickly resorbable carbohydrates should be ingested beforehand to increase the target value and slowly resorbable carbohydrates should be supplemented to stabilize the trend. The amount should be chosen individually and depend on the situation. Classic situations that can result in hypoglycemia are physical activity and alcohol consumption [6].

People who suffer from severe hypoglycemia, who can no longer undergo self-therapy or who are unconscious should not be given oral carbohydrates. In these cases, treatment with glucagon by family members or relatives is possible or professional IV glucose administration. Family members should be trained in the use of glucagon kits [71].

\section{General Nutrition Aspects}

\section{Protein consumption}

\section{Recommendation}

- There are contradictory statements regarding the benefit/ damage for limiting or increasing protein intake as part of a specific diabetes diet. At most, a limitation of the daily protein intake may be useful under a few specific circumstances in existing kidney diseases.

\section{Comment}

Increased protein intake has been scientifically studied in people with type 1 diabetes mellitus, particularly with regard to the acute glycemic response [72]. In this context, reference is made to the recommendation on "insulin requirements for protein- and fat-rich meals".

No evidence has been found in literature that increased protein intake leads to positive or negative effects in people with type $1 \mathrm{di}$ abetes.

A limitation of protein intake has been studied, especially with regard to slowing the progression of diabetic nephropathy.

In general, RCT with intervention times $>6$ months show that protein targets $<0.8 \mathrm{~g} / \mathrm{kg}$ body weight $/ \mathrm{d}$ are rarely met even under study conditions.

In a larger randomized controlled trial, a low-protein diet in people with type 1 or 2 diabetes and nephropathy achieved significant improvements in GFR when compliance was good [73].

In another methodologically well-conducted study, limited protein intake also appeared to slow the progression of diabetic nephropathy, but the effects were not significant. Compliance was also poor in this study and difficult for patients to maintain. Another study showed no difference in the rate of progression of diabetic nephropathy with low normal protein intake $(0.8 \mathrm{~g} / \mathrm{kg}$ body weight, 16 energy\%) vs. high normal protein intake (19 energy\%) [74].

In summary, the evidence for a recommendation for protein reduction - even in diabetic nephropathy - is insufficient. In individual situations and taking into account a high biological value of the proteins, a mild protein restriction may be justified with the aim of inhibiting the progression of diabetic nephropathy. However, this should always be accompanied by professional nutritional advice, since there is a risk of malnutrition especially in advanced stages of renal insufficiency.

\section{General Nutritional Aspects}

\section{Fat intake}

Recommendation

- No specific recommendation can be made for the amount of fat intake in people with T1Dm.

- Effects of the fatty acid composition (fat quality) on other health-promoting aspects may need to be considered separately. However, the fat quality should be in accordance with the recommendations for the general population.

\section{Comment}

For the acute blood glucose response after fat intake, please refer to the chapter "Insulin requirements for protein- and fat-rich meals".

The popularity of low-carbohydrate and thus mostly high-fat diets is high. However, high-fat and low-carbohydrate diets have not been well studied in the treatment of type 1 diabetes. Studies that looked at glycemic outcomes from low-carbohydrate diets were mostly cross-sectional studies without validated nutritional data and without control groups. Participants were highly motivated, self-selected individuals who used intensive insulin management practices, including frequent blood glucose monitoring and 
additional insulin correction with narrow glycemic targets. Therefore, these results are not necessarily transferable to patients with type 1 diabetes in general. Carbohydrate-containing foods such as cereals, fruits and milk are important sources of nutrients. Therefore, low-carbohydrate diets require attention to vitamin and energy intake to avoid micronutrient deficiencies and growth problems in children. Following restrictive diets is a challenge and can affect social normality. People with type 1 diabetes also theoretically have adverse health risks such as diabetic ketoacidosis, hypoglycemia, dyslipidemia and glycogen deficiency [75].

In general, observational studies often associate high fat and low carbohydrate intake with higher BMI [76], but this does not allow the conclusion to be drawn that low fat and high carbohydrate intake is generally recommended. Observational studies often do not address the quality of the fats and carbohydrates ingested, so that it is not taken into account that too many low-value fats and too few complex carbohydrates are usually ingested (see also DDG statement on ADA Recommendations 2019 [5]).

Rather, randomized, controlled intervention studies evaluating the quality of macronutrients should be considered to answer the question of modifying fat intake for people with type 1 diabetes.

\section{Eating Patterns}

\section{Recommendation}

- For people with T1Dm there are a variety of dietary patterns that are suitable. The available evidence is insufficient to recommend a dietary pattern for successful diabetes management.

\section{Comment}

According to the recommendations of the American, Canadian and British diabetes societies, there are a variety of dietary patterns that are suitable for people with diabetes. For example, a diet based on the Mediterranean diet or the Dietary Approaches to Stop Hypertension (DASH) diet could help improve glucose metabolism and weight control and reduce cardiovascular risk factors. However, low-carbohydrate, vegan/vegetarian, plant-based diets or a diet rich in legumes could also be suitable for people with diabetes. Individual preferences, goals and needs should influence the choice of dietary pattern [6-8]. Even specifically for people with T1Dm, the American Diabetes Society says that there is insufficient evidence to make one dietary pattern more recommendable than another [6].

Further evidence from intervention and observational studies for individuals with T1Dm is available on the Mediterranean diet, the DASH diet, the Healthy Eating Index (HEI) or Alternative Healthy Eating Index (AHEI) and the Whole Plant Food Density (WPFD) score. In a cohort study of 118 adults with T1Dm, a Mediterranean diet was associated with a favorable cardiometabolic profile [77], whereas a 6-month intervention study of 28 adults with T1Dm and metabolic syndrome showed no superiority of a Mediterranean vs. low-fat diet in terms of anthropometric and metabolic parameters [78]. In 96 children and adolescents with T1Dm, structured nutritional training for a Mediterranean diet improved the quality of nutrient intake, followed by an improvement in serum lipid levels [79].
In a 5-year longitudinal observational study of 500 children and adolescents with T1Dm, greater adherence to the Mediterranean diet was also associated with better glycemic control and better serum lipid levels [80].

In the SEARCH for Diabetes in Youth Study, it was observed in 2 cross-sectional analyses of 2440 and 1810 adolescents with T1Dm that greater adherence to the DASH diet is inversely associated with hypertension, HbA1c levels and the LDL-HDL ratio [81, 82]. However, in a preliminary controlled intervention study in 16 adolescents with T1Dm, participants on the DASH diet were shown to have increased glycemic variability compared to their usual diet. Adapting the DASH diet to people with diabetes (e. g., $30 \%$ fat instead of $20 \%$ fat) resulted in a glycemic variability comparable to the usual diet, but with lower average blood glucose concentrations and less time in the hyperglycemic range [83].

The role of HEI, an index measuring compliance with U.S. dietary recommendations, was investigated in 4 observational studies in children and adolescents with T1Dm. The studies showed conflicting results regarding the association between the $\mathrm{HEI}$ and glycemic control and no association between the HEl and cardiovascular biomarkers [84-86]. In an observational study of 12 subjects with T1Dm and 75 subjects with T2Dm (no stratified analy$\mathrm{sis})$, greater adherence to the alternate healthy eating index (AHEI) was prospectively associated with improved vascular health [87].

Two cross-sectional studies in adolescents with T1Dm showed that neither the Mediterranean diet, the DASH diet, nor the HEI was associated with selected biomarkers of inflammation, and that only the HEl was inversely related to microalbuminuria, which was no longer significant after adjustment for $\mathrm{HbA} 1 \mathrm{c}$ and systolic blood pressure $[88,89]$.

Greater adherence to whole grains, fruits, vegetables, legumes, nuts, and seeds was associated with better glycemic control in a longitudinal observational study in adolescents with T1Dm [72].

In addition to the evidence for the above described hypothesisbased dietary patterns, 4 cross-sectional and one longitudinal observational study investigated associations between exploratory dietary patterns and metabolic parameters such as glycemic control and blood pressure, inflammatory biomarkers, serum lipid levels, and vascular health parameters in subjects with T1Dm [90-94]. These studies also confirm the conclusion of professional societies that there are a variety of dietary patterns that may be beneficial for people with diabetes, in this case T1Dm [6-8].

\section{Meal Frequency and Meal Timing}

\section{Recommendation}

- If people with T1Dm are overweight, they should avoid irregular eating times, eating late and a time window for daily food intake $>12 \mathrm{~h}$. Instead, they should consume the calories predominantly in the first half of the day, as this can have a beneficial effect on body weight regulation and cardiometabolic risk.

- A recommendation for fasting during the day ( $<3$ meals per day) or modified intermittent fasting on one or more days a week cannot be given. 


\section{Comment}

Irregular food intake, such as skipping meals and snacking behavior, where small portions are often eaten between meals and almost around the clock, is a characteristic of the modern lifestyle [135]. To investigate the influence of frequency and timing of food intake on energy balance and cardiometabolic risk, studies in healthy individuals and subjects with high metabolic and cardiovascular risk are considered below.

A high meal frequency could increase the risk of obesity $[136,137]$, as many small meals lead to less satiety than 3 larger meals with the same energy intake $[138,139]$. In addition, frequent smalls meals compared to fewer meals with the same energy intake resulted in higher liver fat content $[139,140]$.

On the other hand, lower meal frequency, lower caloric intake at breakfast or complete skipping of breakfast was associated with higher weight gain and increased risk of diabetes, heart attack and stroke in prospective long-term studies [141]. Intervention studies of varying durations provide further evidence that skipping breakfast and/or lunch does not have consistently positive effects on body weight regulation and glucose metabolism [135, 142144]. In contrast, skipping the evening meal and eating three meals until early afternoon resulted in improved insulin sensitivity, ß-cell response, blood pressure, and appetite compared to an isocaloric breakfast, lunch, and dinner protocol $[146,148]$.

The influence of the time of mealtime intake on body weight and cardiovascular and metabolic risk is partly explained by circadian differences in metabolism (e. g. with regard to insulin sensitivity). On the other hand, the circadian clock, with the help of clock genes and clock-controlled genes, uses food intake as a timer, so the timing of meals can have an influence on the energy balance and metabolic risk [148]. If food is consumed mainly in the evening or at night, contrary to the natural circadian rhythms, e. g. by skipping breakfast, this leads to lower postprandial thermogenesis and higher postprandial glycemia and insulin response [149]. Consuming low $\mathrm{Gl}$ foods in the morning leads to a greater improvement in glycemia than consuming them in the evening [150].

In addition, a long daily time window in which energy is ingested, independent of the time of food intake, promotes weight gain [135]. A reduction of the daily time window during which food was eaten from more than $14 \mathrm{~h}$ to $10-12 \mathrm{~h}$ led to weight loss with an otherwise unchanged diet [151].

Intermittent fasting is a popular concept for weight loss based on major cycles of dietary restriction, which do not involve skipping a single meal, but instead provide a greatly reduced calorie intake ( 0 to $<25 \%$ of energy requirements) every 2 nd day or $2-3$ times a week. Intermittent fasting results in significant weight loss depending on the frequency of the fasting days, but this is no different from the success of a reduction diet with continuous energy restriction [152-155]. The effects of this negative energy balance lead to a differently pronounced reduction of cardiometabolic risk factors depending on the level of the initial values.

There are worries that intermittent fasting increases the variability of glycemia by increasing the risk of both hypoglycemia and postprandial hyperglycemia $[156,157]$. The lack of superiority of intermittent fasting over continuous, moderate calorie restriction and the lack of studies on the risks and long-term results in sub- jects with T1 Dm make a recommendation for intermittent fasting impossible.

\section{Recommendations for Body Weight}

\section{Recommendation}

- People with T1Dm and normal weight should maintain a normal body weight.

- The study situation is not sufficient to generalize the recommendation for weight reduction to people with type 1 diabetes and excess weight. However, the recommendation for weight reduction may be useful in the presence of concomitant diseases or insulin resistance (e. g. secondary dyslipoproteinemia, high blood pressure, steatohepatitis, etc.).

- The quality of food and possible hypoglycemia should be considered, especially in low-carb diets. Eating disorders may also result.

\section{Comment}

A higher BMI is associated with macrovascular disease and retinopathy in type 1 diabetes [6,7]. There are no evidence-based studies showing an association between weight and glycemic control in type 1 diabetes. However, it can be assumed that weight loss in type $1 \mathrm{di}$ abetes with simultaneous insulin resistance or parallel metabolic syndrome has similar effects to type 2 diabetes [78, 83, 151].

Therefore, weight reduction in obese T1Dm patients is likely to be beneficial in a diet with a low caloric density but high nutritional quality, especially if predominantly abdominal fat distribution is to be reduced.

Very-low-carb diets, formula diets or interval fasting may increase the risk of hypoglycemia and should be used only with close adjustment of the insulin regimen and discussed with the diabetes team.

There is no scientific evidence for the superiority of a nutritional concept (e. g. low-carb or low-fat) in terms of weight loss for T1Dm. Regarding meal frequency, please refer to the text section "Meal frequency and meal timing".

Particularly with the somewhat younger T1Dm patients, the patient desire for weight reduction must be critically weighed against the risk of operating or developing an eating disorder. Female patients are particularly vulnerable compared to male T1Dm patients, [158]. A survey of 34 normal and overweight T1Dm patients showed that weight management and metabolic control measures often contradict each other. This can result in conflict potential and incompliance [159].

Bariatric surgery as a method for weight reduction in patients with T1Dm is to be evaluated critically and must be coordinated with the obesity team and the diabetes team on a case-by-case basis.

Weight reduction in obese T1Dm patients as a side effect of a generally metabolically beneficial diet is likely justifiable, especially if predominantly visceral fat deposits are to be reduced. However, there is insufficient data on this. Very-low-carb diets or interval fasting can increase the risk of hypoglycemia and should therefore only be used with close adaptation of the insulin regimen. 


\section{SCIENTIFIC BACKGROUND}

One study compared a weight loss diet high/fat versus high carb/low fat, with no difference in HbA1c [174].

Observational studies or studies with small numbers of test persons on the consumption of unsaturated fatty acids, n-9 fatty acids or olive oil have not yet provided a reliable basis for recommendations.

In a Spanish observational study, uptake of PUFAs/SFAs $>0.4$ and MUFAs/SFAs $>1.5$ was associated with near-optimal metabolic control goals and a reduction in the risk of diabetic complications [175].

This is supported by another RCT that showed that increased uptake of MUFAs leads to improved glycaemia. A $10 \%$ increase in $n-9$ RCFAs was associated with a $0.64 \%$ improvement in HbA1c [176]. Correspondingly, an RCT showed that acute glycemia was more favorable after olive oil than after butter [177].

\section{Diets for Weight Maintenance}

\section{Recommendation}

- There is no specific recommendation for maintaining weight with T1Dm.

\section{Comment}

Studies on the optimal diet for weight maintenance at T1Dm are not available. The recommendations should be based on the DGE guidelines for healthy individuals, but without defining a specific proportion of macronutrients.

Nutritional intervention studies without weight loss differed in metabolic outcome. With Mediterranean diet $(n=96)$ the lipid profile improved, with low-fat diet $(n=10)$ insulin sensitivity in T1Dm patients was also reduced [160].

Carbohydrate reduction can be safe and with few side effects in individual cases and when used in moderation but without particular benefit (Case Report) [162]. However, the risk of hypoglycemia is increased when short-acting insulins are used with "low carb" [163]. In contrast, other studies also show a reduction in the risk of hypoglycemia with even a severe reduction in carbohydrates ( $<50 \mathrm{~g} ; \mathrm{n}=10.1$ week). Ketonuria as an indicator of insulin deficiency is limited [164]. The effectiveness of emergency glucagon is also reduced due to the lower liver glycogen content [161].

Diets rich in carbohydrates are without advantage or disadvantage when the insulin dose is adjusted ( $n=9,4$ weeks) [165], lower insulin requirements are necessary in the case of a high-fiber variant ( $n=10,4$ weeks) [166], but on the other hand can be associated with a worsening of the metabolic situation - even during intense physical activity ( $n=7,3$ weeks) [167].

Qualitative differences of carbohydrates influence the increase in glucose for T1Dm. Complex carbohydrates should be used to optimize therapy ( $n=12$, one-time acute test of rice vs. pasta varieties) [168]. However, the benefit of a glycemic diet is limited ( $n=11$; 12 weeks, no control group) [169]. As in healthy individuals and
T2Dm, soluble dietary fiber (e.g. guar) does not show the longterm benefit in terms of glycemic control ( $n=8 ; 4$ months) but is still recommended as a component of natural foods for other metabolic reasons [170].

Short-term interventions with the aim of avoiding weight gain, e. g. via VLCD days or short-term intermittent fasting ( $8: 16)$, require individual continuous glycemic control in order to ensure or optimize the suitability of the applied insulin dosing strategy for such days.

\section{Nutritional Aspects in Geriatric Patients}

\section{Recommendation}

- There are no special dietary recommendations for elderly or geriatric persons with T1Dm.

- The goals in nutrition therapy often have a different focus and focus on maintaining independence more than the actual glycemia and, in geriatric patients, on avoiding malnutrition and hypoglycemia.

- The recommendations of a protein intake that meets the needs (at least $1 \mathrm{~g} / \mathrm{kg}$ body weight/d) and the maintenance of a BMI that is up to slightly overweight are basic measures that correspond to the recommendations for the general population and also apply to this group of people.

\section{Comment}

Basically, the nutritional recommendations for older people with T1Dm do not differ from those for older metabolically healthy or younger people with T1Dm. At the same time, the general nutritional recommendations for geriatric patients apply to geriatric patients with T1Dm. Especially in functionally-dependent patients, the consequences of malnutrition in old age are severe and should be focused on in patients with T1Dm. For example, the loss of muscle mass associated with weight loss reinforces the age-related sarcopenia and frailty, thereby promoting disabilities and loss of independence.

The S2k guideline "Diagnosis, therapy and follow-up of diabetes in old age" also contains very detailed recommendations for the general nutritional therapy of elderly people with DM. It makes it clear that therapeutic goals - also with regard to nutrition - can, but need not, often change in older and especially geriatric patients. Functionality and the preservation of independence are in the foreground.

Although an improvement in insulin sensitivity could also be achieved in older people through an intended weight reduction [171], strict dietary restrictions should be avoided in older people who are overweight or obese due to the risk of malnutrition. Dietary restrictions that may limit food intake are potentially harmful and should be avoided. If weight loss is being considered, dietary measures should be combined with physical activity whenever possible, and the focus should be on protein intake to meet the needs. A significant increase in mortality was only found in people over 65 years of age when their body mass index (BMI) exceeded 30 [171].

Restrictions in the consumption of familiar and comfort food lead to a reduction in the subjectively-perceived quality of life. This aspect is particularly important for people in old age. 
The risk of potential malnutrition is present if the reduced food intake continues (approximately $<50 \%$ of the requirement for more than 3 days) or if several risk factors are present at the same time, which either reduce the amount of food or significantly increase the energy and nutrient requirements. The risk of malnutrition can be assessed e. g. by MNA or the corresponding short form (SFMNA), both screening methods are well evaluated [172, 173]. In underweight patients, the causes should be clarified and, if possible, eliminated.

Nutritional therapy should also focus on the prevention of hypoglycemia, with an initial focus on the adjustment of medication.

For further information, especially for persons with diabetes in nursing homes and if artificial nutrition is necessary, please refer to the $\$ 2 \mathrm{k}$ guideline "Diagnosis, therapy and follow-up of diabetes in old age" and the $\$ 3$ guideline "Clinical nutrition in geriatrics" (DDG 2018, DGEM 2013).

Due to the complexity of the often multimorbid geriatric patients, planning and implementation of disease-specific diets should, if necessary, be carried out by a multi-professional team including nutritional expertise.

\section{Nutritional Aspects of Special Foods and Dietary Supplements}

\section{Sugar-sweetened beverages}

Recommendation

- With the exception of treating hypoglycemia, people with T1Dm should minimize their intake of sugar-sweetened beverages.

\section{Comment}

Similar to the evidence for sucrose and fructose intake in people with T1Dm, the data on the intake of sugar-sweetened beverages specifically for this group of patients is also limited, so that recommendations made for the general population and for people with diabetes in general must also be used. According to the current evidence-based guideline of the American Diabetes Association, the intake of sugar-sweetened beverages is strongly discouraged for people with diabetes and water is recommended as often as possible to control blood glucose levels and body weight and reduce the risk of cardiovascular disease and fatty liver (evidence level B) $[4,6]$. The British Diabetes Society rates the evidence on reducing the intake of sugar-sweetened drinks as low but agrees that reducing the consumption of sugar-sweetened drinks reduces cardiovascular risk (Grade 2) [7].

For the general population, a low intake of sugar-sweetened beverages is recommended, among other things because of their high energy content and the resulting risk of obesity. Limiting their intake is recommended because it contributes to an increased micronutrient density and reduced sugar intake [95]. Minimizing their intake and replacing them with water or unsweetened milk or dairy products is also recommended specifically for children and adolescents, regardless of their diabetes status [95]. According to the American Heart Association, the maximum amount for this age group should be around $237 \mathrm{ml}$ per week [95].
For persons with T1Dm, data for children and adolescents are available from 2 observational studies. The Diabetes Autoimmunity Study in the Young observed 1839 children and adolescents with an increased genetic risk for T1Dm over a mean duration of 10.2 years. In study participants with a high-risk HLA genotype, but not in those with a low or medium risk for T1Dm, the progression from islet autoimmunity to T1Dm was associated with an increased intake of sugar-sweetened beverages [96]. Based on cross-sectional analysis of the SEARCH for Diabetes in Youth Study, it was shown that in children and adolescents with manifest T1Dm, a higher (at least one serving per day) intake of sugar-sweetened beverages was associated with higher levels of total cholesterol, LDL cholesterol and plasma triglycerides compared to no intake of sugarsweetened beverages. However, these associations were partially explained by an adjustment for BMI, saturated fat and fiber intake. No association was found between the intake of sugar-sweetened beverages and $\mathrm{HbA} 1 \mathrm{c}$, blood pressure, and HDL cholesterol $[97,98]$. A high intake of sugar-sweetened beverages may therefore have a negative effect on the cardiovascular risk profile in children and adolescents with T1Dm and they should be encouraged to minimize their intake [97].

\section{Alcohol}

Recommendation

- People with T1Dm should limit the amount of alcohol consumed to the amounts recommended for the general population. Moderate, low-risk alcohol consumption is compatible with good metabolic control and diabetes prognosis.

- People with diabetes with a risky alcohol consumption or an alcohol dependency should be informed about the dangers of alcohol, especially with regard to a worsened metabolic control and the risk of secondary diseases.

- It should generally be noted that consumption of larger amounts of alcohol increases the risk of severe hypoglycemia, especially at night, and this risk is reduced by eating during the period of alcohol consumption and raising the target blood glucose level at night.

\section{Comment}

People with diabetes should be advised of the effects of alcohol consumption on blood glucose levels and, if alcohol is consumed, should be encouraged to stick to low-risk consumption amounts. The German Head Office for Addiction Issues e. V. (Deutsche Hauptstelle für Suchtfragen e. V. - DHS) specifies $12 \mathrm{~g}$ alcohol per day for women and $24 \mathrm{~g}$ alcohol per day for men as limits for low-risk consumption. The World Health Organization (WHO) defines a consumption of $10 \mathrm{~g}$ alcohol per day for women and $20 \mathrm{~g}$ alcohol per day for men as low-risk. These amounts also apply to people with type 1 diabetes.

The consumption of alcohol can affect blood glucose counterregulation and thus increase the risk of hypoglycemia. About one in five cases of severe hypoglycemia leading to hospitalization is caused by alcohol consumption. However, the main effect of alcohol is likely to be reduced awareness, which leads to a limited perception of hypoglycemia and prevents those affected from reacting appropriately. A systematic review on this issue is available from 
2018 [178]. A total of 13 studies (RCT and observational studies) were evaluated.

Eight studies reported that ethanol - regardless of whether administered intravenously or orally - was associated with an increased risk of hypoglycemia, which was linked with a decrease in plasma glucose, an impaired counterregulatory response, hypoglycemia perception disorder, and impaired cognitive function.

Five studies could not prove an increased risk of hypoglycemia. None of the studies investigated prevention strategies for ethanolinduced hypoglycemia. Recommendations from 13 diabetes associations were included. All associations recommend only consuming ethanol together with food. The majority of the included studies showed that ethanol consumption increased the risk of hypoglycemia in patients with T1D. However, the evidence for the prevention of ethanol-induced hypoglycemia is scarce and further investigation is needed to provide evidence-based recommendations. Excessive consumption of alcohol interferes with diabetes therapy. Patients with excessive or risky alcohol consumption are less likely to implement therapy recommendations on exercise, diet, medication, blood glucose self-monitoring or regular $\mathrm{HbA} 1 \mathrm{c}$ monitoring. There is a linear relationship: the higher the drinking volume, the less frequently therapy recommendations are implemented.

According to the $52 \mathrm{k}$ guideline Psychosocial factors and diabetes, people with diabetes should be surveyed regularly - at least once a year - for their alcohol consumption, and help should be offered to patients with risky alcohol consumption.

\section{Food supplements}

\section{Recommendation}

- People with T1Dm should cover their nutritional needs with a balanced diet. Routine supplementation with micronutrients is not recommended.

\section{Comment}

The American and Canadian Diabetes Associations summarize the evidence on dietary supplementation in general for people with diabetes as follows: There is no clear evidence that supplementation with vitamins, minerals (e. g., chromium or vitamin D), herbs or spices (e. g., cinnamon or aloe vera) improves metabolic control in people without underlying nutritional deficiencies, and it is not generally recommended to improve glycemic control. Routine supplementation with antioxidants (e. g. vitamin E, C or carotene) is not recommended due to a lack of evidence of efficacy and longterm safety concerns. However, multivitamin supplementation may be necessary in special groups such as pregnant or lactating women, elderly individuals, vegetarians or people on an extremely low-calorie or low-carbohydrate diet [6]. Instead of the general recommendation of routine supplementation, people with diabetes should be encouraged to meet their nutritional needs through a balanced diet [8].

Especially for people with T1Dm, there is scientific evidence for supplementation with vitamin $D$, polyunsaturated fatty acids (PUFAs), chromium, zinc, magnesium and iron.

For supplementation with PUFAs, a systematic review and meta-analysis summarizes data from 7 observational studies in subjects with preclinical and clinical T1Dm [99]. The evidence that
PUFAs protect against preclinical T1Dm was classified as "low" when interventions were combined both during pregnancy and early childhood. However, a sub-analysis based on one study showed that supplementation with n-3 PUFAs in infancy could reduce the risk of preclinical T1Dm. Supplementation with PUFAs was not associated with a risk reduction for clinical T1Dm in children [99].

A cohort study based on 59 children with newly manifested T1Dm and investigating the effects of a 12-month co-supplementation of n-3 PUFAs and vitamin D showed an improvement of surrogate parameters of endogenous insulin secretion in the group of co-supplemented children vs. children supplemented only with vitamin $D$ in a reduction of the nutritive intake of arachidonic acid against the background of a Mediterranean diet [100].

In people with T1Dm, vitamin D is the most commonly studied dietary supplement. Serum concentrations of vitamin D are lower in patients with T1Dm than in controls, but it is still unclear whether vitamin D deficiency is a trigger or consequence of autoimmune disease $[101,102]$. The effects of vitamin D supplementation on the risk of T1Dm in young children have been investigated in 2 meta-analyses and one review, which partly overlap in the included studies [102-104]. There are insufficient studies on whether prenatal supplementation with vitamin $\mathrm{D}$ influences the risk of T1Dm later in life [102, 103].

Observational studies suggest that administration of vitamin D during infancy seems to be associated with a reduced risk of T1Dm [102-104]. A dose-response relationship could be subject to this relationship: a higher vitamin $D$ intake is associated with a lower risk of T1Dm, and the timing of supplementation could also be critical, a lower risk of T1Dm was found with 7-12 months vs. 0 and 6 months of supplementation [104]. Two observational studies also suggest that vitamin $D$ administration in young adults may have a protective effect on the risk of T1Dm [102]. The evidence from intervention studies and randomized controlled trials on the effect of vitamin D supplementation for the treatment of manifest T1Dm is summarized in one systematic review and two additional reviews. Even after that, the results are contradictory, and vitamin D supplementation only shows a protective effect on $\beta$-cell function and glycemic control in some studies $[102,105,106]$. With regard to vitamin $\mathrm{D}$, the current recommendation may be that people with a high genetic risk for T1Dm should avoid vitamin D deficiency by adequate supplementation, but the evidence for the administration of higher doses of vitamin D is insufficient [105].

In a case-cohort study of 257 children with T1Dm, the question was investigated whether iron supplementation during pregnancy and the first 18 months of life is associated with an increased risk of T1Dm in childhood. It was concluded that maternal supplementation with iron is not associated with the risk of T1Dm, but that infantile supplementation shows an inverse association with the risk of T1Dm [107].

Serum concentrations of the trace elements chromium, zinc and magnesium are lower in persons with T1Dm compared to control persons. Observational studies show correlations between lower serum concentrations of these trace elements and poorer glycemic control in subjects with T1Dm, so supplementation may help improve metabolic control. However, further studies are needed to formulate a clear recommendation [108]. For the trace element 
zinc, a further systematic review, a meta-analysis and a review were identified. The former comprises 25 studies, 3 of them with subjects with T1Dm. The 2 case-control studies show an increased zinc concentration in erythrocytes as well as reduced lipid peroxidation after zinc supplementation, and the randomized, double-blind study demonstrates an effect of zinc supplementation on lipid metabolism [109]. A review describes zinc supplementation as a possible measure to improve blood glucose control and lipid metabolism in individuals with T1Dm, but points to the need for further studies due to the conflicting results of existing human studies [110].

In summary, the evidence on the use of dietary supplements in persons with T1Dm is heterogeneous and insufficient. The American and Canadian professional societies do not make a general recommendation on the use of dietary supplements to improve metabolic control. Only the compensation of a nutritive vitamin D deficiency in persons with a high genetic risk for T1Dm could minimize the risk. A balanced diet generally provides an adequate supply of micronutrients.

\section{Probiotics}

\section{Recommendation}

- In view of the current study situation, no recommendation can be made for taking probiotics for the prevention or therapy of type 1 diabetes mellitus.

\section{Comment}

In Germany, probiotics are defined as "defined living microorganisms which enter the intestine in an active form in sufficient quantity and achieve positive health effects" [111].

The Lactobacillus and Bifidobacterium genera are mainly used for the formulation in probiotics. Furthermore, specific lactic acid-producing species of other genera are used, e. g. Enterococcus faecalis, Streptococcus thermophilus or probiotic yeasts (Saccharomyces boulardii). The dose varies between $10^{8}$ and $10^{11}$ colony forming units and the use of the above genera or species is considered safe [112].

Probiotics are classified as dietary supplements in Germany. The manufacturers are legally free to decide on the dose, strains used or any combination (multi-strain preparations) as well as possible additives (vitamins, prebiotics, etc.). Health claims or claims referring to the reduction of a disease risk are not permitted for probiotics, except for the improvement of lactose intolerance and the reduction of infections (EC 1924/2006).

The intestinal microbiome and the immune system are closely intertwined [113]. The immunomodulating effect of the intestinal microbiota or its metabolites, such as short-chain fatty acids, especially butyrate, is shown, among other things, by their effect on regulatory T cells $[114,115]$, the inflammatory status [114] and the function of the intestinal barrier [116]. In several studies of volunteers with diagnosed type 1 diabetes, microbial diversity was found to be lower than in healthy volunteers [117-119]. In the period immediately before or at the onset of beta-cell autoimmunity, a higher proportion of Bacteroides was repeatedly observed in the subjects compared to the control group [120-123]. The lipopolysaccharide (LPS) produced by Bacteroides showed an immunosuppressive effect (measured by TLR4 and NF-KB activity) in children prone to autoimmune disease [124]. Current studies also suggest an association between reduced production of short-chain fatty acids, especially butyrate, by the microbiome and an increased risk of type 1 diabetes $[117,124,125]$. This leads to the hypothesis that intestinal microbial disorders are associated with the pathogenesis of beta-cell autoimmunity and type 1 diabetes mellitus [126, 127].

To test the preventive effect of probiotics on the development of beta-cell autoimmunity, studies were conducted in genetically predisposed children before the onset of autoimmunity, primarily in infancy and early childhood $[128,129]$. In the prospective, longitudinal, multicenter cohort "Environmental Determinants of Diabetes in the Young” (TEDDY), probiotic intake pre- and postnatally was assessed in 7473 infants/children with genetic predisposition to type 1 diabetes. Early infant (0-27 days postnatal) probiotic intake may reduce the risk of beta-cell autoimmunity in children with high risk genotype (HLA-DR3/4) [128]. On the other hand, in a double-blind, randomized, controlled trial, the administration of probiotics in infancy could not be associated with the development of beta-cell autoimmunity [129].

In addition to preventive research on beta-cell autoimmunity, there are also studies on the effects of probiotics in already manifest type 1 diabetes. An eight-week supplementation of synbiotics (Lactobacillus sporogenes GBI-30 with maltodextrin and fructooligosaccharides) in 50 subjects (4-18 years old) with diagnosed type 1 diabetes resulted in an improvement in fasting blood glucose, HbA1c, hs-CRP and TAC (total antioxidant capacity) [179].

Another intervention study was conducted to determine whether a three-month supplementation with Lactobacillus rhamnosus GG leads to an increased immune function in type 1 diabetes patients following an influenza vaccination. No improvement in the humoral immune response was observed, but an anti-inflammatory effect was induced by supplementation [130].

The following points must also be considered when supplementing with probiotics: on the one hand, probiotics can exhibit antibiotic resistance in mobile genetic elements that can be transferred to other, possibly pathogenic bacteria through interbacterial exchange [131]. The investigation of various commerciallyavailable probiotics showed that the probiotic bacteria tested were resistant to several different broad-spectrum antibiotics [132]. On the other hand, in rare cases, a weakened intestinal barrier function in critically-ill patients can lead to a translocation of the supplemented microorganisms [133]. In addition, possible side effects are not recorded in many studies in which the use of probiotics is tested.

Finding a causal relationship between intestinal microbiota and the pathogenesis of beta-cell autoimmunity is proving difficult. On the one hand, this is due to the high heterogeneity of the genera or species of probiotics used, the study populations as well as the study parameters; on the other hand, to the individuality and complexity of the microbiome. New findings also show that the administration of probiotics can significantly influence the efficacy of a probiotic by making it resistant to colonization by individuals, strains and regions, depending on the indigenous microbiome [134]. Overall, there is evidence to suggest that probiotic intake may be helpful in the prevention or therapy of islet autoimmunity and type 1 diabetes. However, in the current study situation, no recommendation can yet be made to take probiotics for the prevention or therapy of type 1 diabetes mellitus. 


\section{Conflict of Interest}

The authors declare that there is no conflict of interest.

\section{References}

[1] Toeller M. Evidence of Nutrition in the Treatment and Prevention of Diabetes Mellitus. Current Nutritional Medicine 2005; 30: 197-203

[2] Beck J, Greenwood DA, Blanton L et al. 2017; National Standards for Diabetes Self-Management Education and Support. Diabetes Educ 2020; 46: 46-61

[3] Beck J, Greenwood DA, Blanton L et al. 2017; National Standards for Diabetes Self-Management Education and Support. Diabetes Care 2017; 40: 1409-1419

[4] Evert AB, Dennison M, Gardner CD et al. Nutrition Therapy for Adults With Diabetes or Prediabetes: A Consensus Report. Diabetes Care 2019; 42: 731-754

[5] DDG https://www.deutsche-diabetes-gesellschaft.de/fileadmin/ user_upload/06_Gesundheitspolitik/01_Stellungnahmen/2019/20190927_Ausschuss_Ernaehrung_DDG_Stellungnahme_ zu_den_ADA_Ernaehrungsempfehlungen.pdf

[6] American Diabetes Association 5. Facilitating Behavior Change and Well-being to Improve Health Outcomes: Standards of Medical Care in Diabetes-2020. Diabetes Care 2020; 43: S48-S65

[7] Dyson PA, Twenefour D, Breen C et al. Diabetes UK evidence-based nutrition guidelines for the prevention and management of diabetes. Diabet Med 2018; 35: 541-547

[8] Dworatzek PD, Arcudi K, Gougeon R et al. Nutrition therapy. Can ] Diabetes 2013; 37: S45-S55

[9] Kröger J, Siegmund T, Schubert-Olesen O et al. AGP Primer Nutrition. Analysing postprandial glucose courses with CGM. Mainz: Kirchheim + Co; 2020

[10] Scavone G, Manto A, Pitocco D et al. Effect of Carbohydrate Counting and Medical Nutritional Therapy on Glycaemic Control in Type 1 Diabetic Subjects: A Pilot Study. Diabet Med 2010; 27: 477-479

[11] Smart CE, King BR, McElduff $P$ et al. In children using intensive insulin therapy, a $20 \mathrm{~g}$ variation in carbohydrate amount signigicantly impacts on postprandial glycaemia. Diabet Med 2012; 29: e21-e24

[12] Bell KJ, King BR, Shafat A et al. The Relationship Between Carbohydrate and the Mealtime Insulin Dose in Type 1 Diabetes. J Diabetes Complications 2015; 29: 1323-1329

[13] Smart CE, Ross K et al. Can children with Type 1 diabetes and their caregivers estimate the carbohydrate conent of meals and snacks. Diabet Med 2010; 27: 348-353

[14] Atkinson FS, Foster-Powell K, Brand-Miller JC. International tables of glycemic index and glycemic load values: 2008. Diabetes Care 2008; 31: 2281-2283

[15] Chantelau EA. Quantification of the blood glucose effect of foods: the glycaemic index (Chapter 14.4.2).In: Berger M, (Ed.) Diabetes mellitus. 2nd ed.Munich Jena: Urban \& Fischer; 2000: 158-163

[16] Strohm D. Glycemic index and glycemic load - a relevant concept for the nutritional practice of healthy people? Scientific statement of the DGE. Nutrition review 2013; 60: M26-M38

[17] Chantelau E, Kronsbein P, Kempf $U$ et al. Investigation of the meal-related insulin requirement of lentils and potatoes in patients with type I diabetes mellitus. Current nutritional medicine 1989; 14: 175-178

[18] Lafrance L, Rabasa-Lhoret R, Poisson D et al. Effects of different glycaemic index foods and dietary fibre intake on glycaemic control in type 1 diabetic patients on intensive insulin therapy. Diabet Med 1998; 15: 972-978
[19] Parillo M, Annuzzi G, Rivellese AA et al. Effects of meals with different glycaemic index on postprandial blood glucose response in patients with Type 1 diabetes treated with continuous subcutaneous insulin infusion. Diabet Med 2011; 28: 227-229

[20] Chantelau E, Spraul M, Kunze K et al. Effects of the glycaemic index of dietary carbohydrates on prandial glycaemia and insulin therapy in type I diabetes mellitus. Diabetes Res Clin Pract 1986; 2: 35-41

[21] Fontvieille AM, Acosta M, Rizkalla SW et al. A moderate switch from high to low glycaemic-index foods for 3 weeks improves the metabolic control of Type I (IDDM) diabetic subjects. Diabetes Nutr Metab 1988; 1: 139-143

[22] Thomas DE, Elliott E]. The use of low-glycaemic index diets in diabetes control. Br J Nutr 2010; 104: 797-802

[23] Giacco R, Parillo M, Rivellese AA et al. Long-term dietary treatment with increased amounts of fiber-rich low-glycemic index natural foods improves blood glucose control and reduces the number of hypoglycemic events in type 1 diabetic patients. Diabetes Care 2000; 23: 1461-1466

[24] Fontvieille AM, Rizkalla SW, Penfornis A et al. The use of low glycaemic index foods improves metabolic control of diabetic patients over five weeks. Diabet Med 1992; 9: 444-450

[25] Vega-López S, Venn BJ, Slavin JL. Relevance of the Glycemic Index and Glycemic Load for Body Weight, Diabetes, and Cardiovascular Disease. Nutrients 2018; 10

[26] Nansel TR, Gellar L, McGill A. Effect of varying glycemic index meals on blood glucose control assessed with continuous glucose monitoring in youth with type 1 diabetes on basal-bolus insulin regimens. Diabetes Care 2008; 31: 695-697

[27] Ryan RL, King BR, Anderson DG et al. Influence of and optimal insulin therapy for a low-glycemic index meal in children with type 1 diabetes receiving intensive insulin therapy. Diabetes Care 2008; 31 : $1485-1490$

[28] Collier GR, Giudici S, Kalmusky J et al. Low glycaemic index starchy foods improve glucose control and lower serum cholesterol in diabetic children. Diabetes Nutr Metab 1988; 1: 11-19

[29] Gilbertson HR, Brand-Miller JC, Thorburn AW et al. The effect of flexible low glycemic index dietary advice versus measured carbohydrate exchange diets on glycemic control in children with type 1 diabetes. Diabetes Care 2001; 24: 1137-1143

[30] Pańkowska E, Błazik M, Groele L. Does the fat-protein meal increase postprandial glucose level in type 1 diabetes patients on insulin pump: the conclusion of a randomized study. Diabetes Technol Ther 2012; 14: 16-22

[31] Winiger $G$, Keller $U$, Laager R et al. Protein content of the evening meal and nocturnal plasma glucose regulation in type-I diabetic subjects. Horm Res 1995; 44: 101-104

[32] Smart CEM, Evans M, O'Connell SM et al. Both dietary protein and fat increase postprandial glucose excursions in children with type 1 diabe-tes, and the effect is additive. Diabetes Care 2013; 36: 3897-3902

[33] Pańkowska E, Błazik M. Bolus calculator with nutrition database software, a new concept of prandial insulin programming for pump users. J Diabetes Sci Technol 2010; 4: 571-576

[34] Hermann $\mathrm{K}$ et al. Studies on the effectiveness and safety of additional insulin administration for meals with high fat and protein content in patients with type 1 diabetes mellitus. Within the scope of a doctoral thesis. 2019;

[35] Eschenburger $\mathrm{S}$, Tombek $\mathrm{A}$ et al. investigation of a nut meal as a late meal under consideration of a continuous glucose measurement. In the context of a master thesis. not published 2020;

[36] Krems C, Walter C, Heuer T et al. National Consumption Study II - Food Consumption and Nutritional Supply based on 24h-Recalls. Max Rubner Institute. 2013; 
[37] Hakola L, Miettinen ME, Syrjälä E et al. Association of Cereal, Gluten, and Dietary Fiber Intake With Islet Autoimmunity and Type 1 Diabetes. JAMA Pediatr. 2019

[38] de Carvalho CM, Gross LA, de Azevedo MJ et al. Dietary Fiber Intake (Supplemental or Dietary Pattern Rich in Fiber) and Diabetic Kidney Disease: A Systematic Review of Clinical Trials. Nutrients 2019; 11

[39] Beretta MV, Bernaud FR, Nascimento C et al. Higher fiber intake is associated with lower blood pressure levels in patients with type 1 diabetes. Arch Endocrinol Metab 2018; 62: 47-54

[40] Bernaud FSR, Beretta MV, do Nascimento C et al Fiber intake and inflammation in type 1 diabetes. Diabetol Metab Syndr 2014; 6: 66

[41] Mottalib A, Kasetty M, Mar JY et al. Weight Management in Patients with Type 1 Diabetes and Obesity. Curr Diab Rep 2017; 17

[42] Basu A, Alman AC, Snell-Bergeon JK. Dietary fiber intake and glycemic control: coronary artery calcification in type 1 diabetes (CACTI) study. Nutr J 2019; 18: 23

[43] Ho J, Nicolucci AC, Virtanen H et al. Effect of Prebiotic on Microbiota, Intestinal Permeability, and Glycemic Control in Children With Type 1 Diabetes. J Clin Endocrinol Metab 2019; 104: 4427-4440

[44] European Commission. Health Promotion and Disease Prevention Knowledge Gateway. Sugars and Sweeteners (01.02.2020). Im Internet https://ec.europa.eu/jrc/en/health-knowledge-gateway/ promotion-prevention/nutrition/sugars-sweeteners

[45] Franz M], Powers MA, Leontos C et al. The Evidence for Medical Nutrition Therapy for Type 1 and Type 2 Diabetes in Adults. J Am Diet Assoc 2010; 110: 1852-1889

[46] Rippe JM, Angelopoulos TJ. Sugars, obesity, and cardiovascular disease: results from recent randomized control trials. Eur J Nutr 2016; 55: 45-53

[47] Souto DL, Zajdenverg L, Rodacki M et al. Does sucrose intake affect antropometric variables, glycemia, lipemia and C-reactive protein in subjects with type 1 diabetes?: a controlled-trial. Diabetol Metab Syndr 2013; 5: 67

[48] Rippe JM, Angelopoulos T]. Sugars, obesity, and cardiovascular disease: results from recent randomized control trials. Eur J Nutr 2016; 55: 45-53

[49] Cozma Al, Sievenpiper JL, de Souza RJ et al. Effect of Fructose on Glycemic Control in Diabetes: A systematic review and meta-analysis of controlled feeding trials. Diabetes Care 2012; 35: 1611-1620

[50] Evans RA, Frese M, Romero J et al. Fructose replacement of glucose or sucrose in food or beverages lowers postprandial glucose and insulin without raising triglycerides: a systematic review and meta-analysis. Am J Clin Nutr 2017; 106: 506-518

[51] Sievenpiper JL, Carleton AJ, Chatha $S$ et al. Heterogeneous Effects of Fructose on Blood Lipids in Individuals With Type 2 Diabetes: Systematic review and meta-analysis of experimental trials in humans. Diabetes Care 2009; 32: 1930-1937

[52] Souto DL, Lima ÉS, Dantas JR et al. Postprandial metabolic effects of fructose and glucose in type 1 diabetes patients: a pilot randomized crossover clinical trial. Archives of Endocrinology and Metabolism 2019; 63: 376-384

[53] European Commission Health Promotion and Disease Prevention Knowledge Gateway: Sugars and Sweeteners. 2020;Available from https://ec.europa.eu/jrc/en/health-knowledge-gateway/promotionprevention/nutrition/sugars-sweeteners

[54] American Diabetes Association (ADA) 4. Foundations of Care: Education, Nutrition, Physical Activity, Smoking Cessation, Psychosocial Care, and Immunization. Diabetes Care 2014; 38: 20-31

[55] Evert AB, Dennison M, Gardner CD et al. Nutrition Therapy for Adults With Diabetes or Prediabetes: A Consensus Report. Diabetes Care 2019; 42: 731-754
[56] Sjöblad S. Could the high consumption of high glycaemic index carbohydrates and sugars, associated with the nutritional transition to the Western type of diet, be the common cause of the obesity epidemic and the worldwide increasing incidences of Type 1 and Type 2 diabetes? Medical Hypotheses 2019; 125: 41-50

[57] Lamb MM, Frederiksen B, Seifert JA et al. Sugar intake is associated with progression from islet autoimmunity to type 1 diabetes: the Diabetes Autoimmunity Study in the Young. Diabetologia 2015; 58: 2027-2034

[58] Couch SC, Crandell JL, Shah AS et al. Fructose intake and cardiovascular risk factors in youth with type 1 diabetes: SEARCH for diabetes in youth study. Diabetes Research and Clinical Practice 2013; 100 : 265-271

[59] Nansel TR, Lipsky LM, Liu A. Greater diet quality is associated with more optimal glycemic control in a longitudinal study of youth with type 1 diabetes. The American Journal of Clinical Nutrition 2016; 104: 81-87

[60] EFSA completes full risk assessment on aspartame and concludes that it is safe at current exposure levels, 10.12.2013. On the internet (as of 02.08.2020) https://www.efsa.europa.eu/en/press/news/131210

[61] Toews I, Lohner S, Küllenberg de Gaudry D et al. Association between intake of non-sugar sweeteners and health outcomes: systematic review and meta-analyses of randomised and non-randomised controlled trials and observational studies. BMJ 2019; 364: k4718

[62] Sylvetsky AC, Rother KI. Nonnutritive Sweeteners in Weight Management and Chronic Disease: A Review. Obesity (Silver Spring) 2018; 26: 635-640

[63] Ashwell M, Gibson S, Bellisle F et al. Expert consensus on low-calorie sweeteners: facts, research gaps and suggested actions. Nutr Res Rev 2020; $1-10$

[64] Romo-Romo A, Aguilar-Salinas CA, Brito-Córdova GX et al. Effects of the Non-Nutritive Sweeteners on Glucose Metabolism and Appetite Regulating Hormones: Systematic Review of Observational Prospective Studies and Clinical Trials. PLoS ONE 2016; 11: e0161264

[65] Rogers P], Hogenkamp PS, de Graaf C et al. Does low-energy sweetener consumption affect energy intake and body weight? A systematic review, including meta-analyses, of the evidence from human and animal studies. Int J Obes (Lond) 2016; 40: 381-394

[66] Fantino M, Fantino A, Matray M et al. Beverages containing low energy sweeteners do not differ from water in their effects on appetite, energy intake and food choices in healthy, non-obese French adults. Appetite 2018; 125: 557-565

[67] Bellisle F. Intense Sweeteners, Appetite for the Sweet Taste, and Relationship to Weight Management. Curr Obes Rep 2015; 4: 106-110

[68] Lobach AR, Roberts A, Rowland IR. Assessing the in vivo data on low/ no-calorie sweeteners and the gut microbiota. Food Chem Toxicol 2019; 124: 385-399

[69] Ahmad SY, Azad MB, Friel J et al. Recent evidence for the effects of nonnutritive sweeteners on glycaemic control. Curr Opin Clin Nutr Metab Care 2019; 22: 278-283

[70] Hunter SR, Reister E], Cheon E et al. Low Calorie Sweeteners Differ in Their Physiological Effects in Humans. Nutrients 2019; 11

[71] German Diabetes Society https://www.awmf.org/uploads/tx_ szleitlinien/057-013|_S3-TherapyType1Diabetes_2018-08.pdf

[72] Nansel TR, Lipsky LM, Liu A. Greater diet quality is associated with more optimal glycemic control in a longitudinal study of youth with type 1 diabetes. Am J Clin Nutr 2016; 104: 81-87

[73] Nezu U, Kamiyama H, Kondo $\mathrm{Y}$ et al. Effect of low-protein diet on kidney function in diabetic nephropathy: meta-analysis of randomised controlled trials. BMJ open 2013; 3

[74] Dussol B, lovanna C, Raccah D et al. A randomized trial of low-protein diet in type 1 and in type 2 diabetes mellitus patients with incipient and overt nephropathy. J Ren Nutr 2005; 15: 398-406 
[75] Seckold R, Fisher E, de Bock M et al. The ups and downs of low-carbohydrate diets in the management of Type 1 diabetes: a review of clinical outcomes. Diabet Med 2019; 36: 326-334

[76] Donaghue KC, Pena MM, Chan AKF et al. Beneficial effects of increasing monounsaturated fat intake in adolescents with type 1 diabetes. Diabetes Res Clin Pract 2000; 48: 193-199

[77] Gingras V, Leroux C, Desjardins K et al. Association between Cardiometabolic Profile and Dietary Characteristics among Adults with Type 1 Diabetes Mellitus. J Acad Nutr Diet 2015; 115: 1965-1974

[78] Fortin A, Rabasa-Lhoret R, Lemieux S et al. Comparison of a Mediterranean to a low-fat diet intervention in adults with type 1 diabetes and metabolic syndrome: A 6-month randomized trial. Nutr Metab Cardiovasc Dis 2018; 28: 1275-1284

[79] Cadario F, Prodam F, Pasqualicchio $S$ et al. Lipid profile and nutritional intake in children and adolescents with Type 1 diabetes improve after a structured dietician training to a Mediterranean-style diet. J Endocrinol Invest 2012; 35: 160-168

[80] Zhong VW, Lamichhane AP, Crandell JL et al. Association of adherence to a Mediterranean diet with glycemic control and cardiovascular risk factors in youth with type I diabetes: the SEARCH Nutrition Ancillary Study. Eur ] Clin Nutr 2016; 70: 802-807

[81] Günther ALB, Liese AD, Bell RA et al. Association between the dietary approaches to hypertension diet and hypertension in youth with diabetes mellitus. Hypertension 2009; 53: 6-12

[82] Liese AD, Bortsov A, Günther ALB et al. Association of DASH diet with cardiovascular risk factors in youth with diabetes mellitus: the SEARCH for Diabetes in Youth study. Circulation 2011; 123: 1410-1417

[83] Peairs AD, Shah AS, Summer $S$ et al. Effects of the dietary approaches to stop hypertension (DASH) diet on glucose variability in youth with Type 1 diabetes. Diabetes Manag (Lond) 2017; 7: 383-391

[84] Nansel TR, Haynie DL, Lipsky LM et al. Multiple indicators of poor diet quality in children and adolescents with type 1 diabetes are associated with higher body mass index percentile but not glycemic control. J Acad Nutr Diet 2012; 112: 1728-1735

[85] Powers MA, Gal RL, Connor CG et al. Eating patterns and food intake of persons with type 1 diabetes within the T1D exchange. Diabetes Res Clin Pract 2018; 141: 217-228

[86] Sanjeevi N, Lipsky LM, Nansel TR. Cardiovascular Biomarkers in Association with Dietary Intake in a Longitudinal Study of Youth with Type 1 Diabetes. Nutrients 2018; 10

[87] Petersen KS, Keogh JB, Lister NB et al. Dietary quality and carotid intima media thickness in type 1 and type 2 diabetes: Follow-up of a randomised controlled trial. Nutr Metab Cardiovasc Dis 2018; 28 : 830-838

[88] Costacou T, Crandell J, Kahkoska AR et al. Dietary Patterns Over Time and Microalbuminuria in Youth and Young Adults With Type 1 Diabetes: The SEARCH Nutrition Ancillary Study. Diabetes Care 2018; 41: 1615-1622

[89] Liese AD, Ma X, Ma X et al. Dietary quality and markers of inflammation: No association in youth with type 1 diabetes. J Diabetes Complicat 2018; 32: 179-184

[90] Ahola A], Freese R, Mäkimattila $S$ et al. Dietary patterns are associated with various vascular health markers and complications in type 1 diabetes. J Diabetes Complicat 2016; 30: 1144-1150

[91] Ahola AJ, Gordin D, Forsblom C et al. Association between diet and measures of arterial stiffness in type 1 diabetes - Focus on dietary patterns and macronutrient substitutions. Nutr Metab Cardiovasc Dis 2018; 28: 1166-1172

[92] Jaacks LM, Crandell J, Mendez MA et al. Dietary patterns associated with $\mathrm{HbA} 1 \mathrm{c}$ and $\mathrm{LDL}$ cholesterol among individuals with type 1 diabetes in China. J Diabetes Complicat 2015; 29: 343-349
[93] Lamichhane AP, Liese AD, Urbina EM et al. Associations of dietary intake patterns identified using reduced rank regression with markers of arterial stiffness among youth with type 1 diabetes. Eur J Clin Nutr 2014; 68: 1327-1333

[94] van Bussel BCT, Soedamah-Muthu SS, Henry RMA et al. Unhealthy dietary patterns associated with inflammation and endothelial dysfunction in type 1 diabetes: the EURODIAB study. Nutr Metab Cardiovasc Dis 2013; 23: 758-764

[95] European Commission Health Promotion and Disease Prevention Knowledge Gateway: Sugars and Sweeteners. https://ec.europa.eu/ jrc/en/health-knowledge-gateway/promotion-prevention/nutrition/ su gars-sweeteners

[96] Lamb MM, Frederiksen B, Seifert JA et al. Sugar intake is associated with progression from islet autoimmunity to type 1 diabetes: the Diabetes Autoimmunity Study in the Young. Diabetologia 2015; 58: 2027-2034

[97] Bortsov AV, Liese AD, Bell RA et al. Sugar-sweetened and diet beverage consumption is associated with cardiovascular risk factor profile in youth with type 1 diabetes. Acta Diabetol 2011; 48: 275-282

[98] Liese AD, Crandell JL, Tooze JA et al. Sugar-sweetened beverage intake and cardiovascular risk factor profile in youth with type 1 diabetes: application of measurement error methodology in the SEARCH Nutrition Ancillary Study. Br J Nutr 2015; 114: 430-438

[99] Liu X, Zhang Y, Wu H et al. Intake of polyunsaturated fatty acids and risk of preclinical and clinical type 1 diabetes in children-a systematic review and meta-analysis. Eur J Clin Nutr 2019; 73: 1-8

[100] Cadario F, Pozzi E, Rizzollo S et al. Vitamin D and $\omega$-3 Supplementations in Mediterranean Diet During the 1st Year of Overt Type 1 Diabetes: A Cohort Study. Nutrients 2019; 11

[101] Antonucci R, Locci C, Clemente MG et al. Vitamin D deficiency in childhood: old lessons and current challenges. J Pediatr Endocrinol Metab 2018; 31: 247-260

[102] Rak K, Bronkowska M. Immunomodulatory Effect of Vitamin D and Its Potential Role in the Prevention and Treatment of Type 1 Diabetes Mellitus-A Narrative Review. Molecules 2018; 24

[103] Dong JY, Zhang WG, Chen JJ et al. Vitamin D intake and risk of type 1 diabetes: a meta-analysis of observational studies. Nutrients 2013; 5: 3551-3562

[104] Zipitis CS, Akobeng AK. Vitamin D supplementation in early childhood and risk of type 1 diabetes: a systematic review and meta-analysis. Arch Dis Child 2008; 93: 512-517

[105] Altieri B, Muscogiuri G, Barrea L et al. Does vitamin D play a role in autoimmune endocrine disorders? A proof of concept. Rev Endocr Metab Disord 2017; 18: 335-346

[106] Gregoriou E, Mamais I, Tzanetakou I et al. The Effects of Vitamin D Supplementation in Newly Diagnosed Type 1 Diabetes Patients: Systematic Review of Randomized Controlled Trials. Rev Diabet Stud 2017; 14: 260-268

[107] Thorsen SU, Halldorsson TI, Bjerregaard AA et al. Maternal and Early Life Iron Intake and Risk of Childhood Type 1 Diabetes: A Danish Case-Cohort Study. Nutrients 2019; 11

[108] Lin CC, Huang YL. Chromium, zinc and magnesium status in type 1 diabetes. Curr Opin Clin Nutr Metab Care 2015; 18: 588-592

[109] Jayawardena R, Ranasinghe P, Galappatthy P et al. Effects of zinc supplementation on diabetes mellitus: a systematic review and meta-analysis. Diabetol Metab Syndr 2012; 4: 13

[110] Chimienti F. Zinc, pancreatic islet cell function and diabetes: new insights into an old story. Nutr Res Rev 2013; 26: 1-11

[111] BgVV working group https://mobil.bfr.bund.de/cm/343/probiot.pdf

[112] de Vrese M. Microbiology, effect and safety of probiotics. monthly journal pediatrics 2008; 156: 1063-1069 
[113] Zheng D, Liwinski T, Elinav E. Interaction between microbiota and immunity in health and disease. Cell Res 2020; 30: 492-506

[114] Arpaia N, Campbell C, Fan X et al. Metabolites produced by commensal bacteria promote peripheral regulatory T-cell generation. Nature 2013; 504: 451-455

[115] Smith PM, Howitt MR, Panikov N et al. The microbial metabolites, short-chain fatty acids, regulate colonic Treg cell homeostasis. Science 2013; 341: 569-573

[116] Bosi E, Molteni L, Radaelli MG et al. Increased intestinal permeability precedes clinical onset of type 1 diabetes. Diabetologia 2006; 49: 2824-2827

[117] Brown CT, Davis-Richardson AG, Giongo A et al. Gut microbiome metagenomics analysis suggests a functional model for the development of autoimmunity for type 1 diabetes. PLoS ONE 2011; 6: e25792

[118] de Goffau MC, Luopajärvi K, Knip M et al. Fecal microbiota composition differs between children with $\beta$-cell autoimmunity and those without. Diabetes 2013; 62: 1238-1244

[119] Kostic AD, Gevers D, Siljander $\mathrm{H}$ et al. The dynamics of the human infant gut microbiome in development and in progression toward type 1 diabetes. Cell Host Microbe 2015; 17: 260-273

[120] Davis-Richardson AG, Ardissone AN, Dias R et al. Bacteroides dorei dominates gut microbiome prior to autoimmunity in Finnish children at high risk for type 1 diabetes. Front Microbiol 2014; 5: 678

[121] de Goffau MC, Fuentes S, van den Bogert B et al. Aberrant gut microbiota composition at the onset of type 1 diabetes in young children. Diabetologia 2014; 57: 1569-1577

[122] Alkanani AK, Hara N, Gottlieb PA et al. Alterations in Intestinal Microbiota Correlate With Susceptibility to Type 1 Diabetes. Diabetes 2015; 64: 3510-3520

[123] Vatanen T, Franzosa EA, Schwager $R$ et al. The human gut microbiome in early-onset type 1 diabetes from the TEDDY study. Nature 2018; 562: 589-594

[124] Vatanen T, Kostic AD, d'Hennezel E et al. Variation in Microbiome LPS Immunogenicity Contributes to Autoimmunity in Humans. Cell 2016; 165: 842-853

[125] Vatanen T, Franzosa EA, Schwager R et al. The human gut microbiome in early-onset type 1 diabetes from the TEDDY study. Nature 2018; 562: 589-594

[126] Gavin PG, Hamilton-Williams EE. The gut microbiota in type 1 diabetes: friend or foe? Curr Opin Endocrinol Diabetes Obes 2019; 26: $207-212$

[127] Mishra SP, Wang S, Nagpal R et al. Probiotics and Prebiotics for the Amelioration of Type 1 Diabetes: Present and Future Perspectives. Microorganisms 2019; 7

[128] Uusitalo U, Liu X, Yang J et al. Association of Early Exposure of Probiotics and Islet Autoimmunity in the TEDDY Study. JAMA Pediatr 2016; 170: 20-28

[129] Savilahti E, Härkönen T, Savilahti EM et al. Probiotic intervention in infancy is not associated with development of beta cell autoimmunity and type 1 diabetes. Diabetologia 2018; 61: 2668-2670

[130] Bianchini S, Orabona C, Camilloni B et al. Effects of probiotic administration on immune responses of children and adolescents with type 1 diabetes to a quadrivalent inactivated influenza vaccine. Hum Vaccin Immunother 2020; 16: 86-94

[131] Zheng M, Zhang R, Tian X et al. Assessing the Risk of Probiotic Dietary Supplements in the Context of Antibiotic Resistance. Front Microbiol 2017; 8: 908

[132] Wong A, Ngu DYS, Dan LA et al. Detection of antibiotic resistance in probiotics of dietary supplements. Nutr J 2015; 14: 95

[133] Singhi SC, Kumar S. Probiotics in critically ill children. F1000Res 2016; 5
[134] Zmora N, Zilberman-Schapira G, Suez J et al. Personalized Gut Mucosal Colonization Resistance to Empiric Probiotics Is Associated with Unique Host and Microbiome Features. Cell 2018; 174: 1388-1405.e21

[135] Gill S, Panda S. A Smartphone App Reveals Erratic Diurnal Eating Patterns in Humans that Can Be Modulated for Health Benefits. Cell Metab 2015; 22: 789-798

[136] Kahleova H, Lloren Jl, Mashchak A et al. Meal Frequency and Timing Are Associated with Changes in Body Mass Index in Adventist Health Study 2. J Nutr 2017; 147: 1722-1728

[137] McCrory MA, Howarth NC, Roberts SB et al. Eating frequency and energy regulation in free-living adults consuming self-selected diets. J Nutr 2011; 141: 148-153

[138] Ohkawara K, Cornier MA, Kohrt WM et al. Effects of increased meal frequency on fat oxidation and perceived hunger. Obesity (Silver Spring) 2013; 21:336-343

[139] Kahleova $\mathrm{H}$, Belinova L, Malinska $\mathrm{H}$ et al. Eating two larger meals a day (breakfast and lunch) is more effective than six smaller meals in a reduced-energy regimen for patients with type 2 diabetes: a randomised crossover study. Diabetologia 2014; 57: 1552-1560

[140] Koopman KE, Caan MWA, Nederveen A] et al. Hypercaloric diets with increased meal frequency, but not meal size, increase intrahepatic triglycerides: a randomized controlled trial. Hepatology 2014; 60: 545-553

[141] St-Onge MP, Ard J, Baskin ML et al. Meal Timing and Frequency: Implications for Cardiovascular Disease Prevention: A Scientific Statement From the American Heart Association. Circulation 2017; 135: e96-e121

[142] Stote KS, Baer DJ, Spears K et al. A controlled trial of reduced meal frequency without caloric restriction in healthy, normal-weight, middle-aged adults. Am J Clin Nutr 2007; 85: 981-988

[143] Tinsley GM, Forsse JS, Butler NK et al. Time-restricted feeding in young men performing resistance training: A randomized controlled trial. Eur J Sport Sci 2017; 17: 200-207

[144] Moro T, Tinsley G, Bianco A et al. Effects of eight weeks of timerestricted feeding (16/8) on basal metabolism, maximal strength, body composition, inflammation, and cardiovascular risk factors in resistance-trained males. J Transl Med 2016; 14: 290

[145] Nas A, Mirza N, Hägele F et al. Impact of breakfast skipping compared with dinner skipping on regulation of energy balance and metabolic risk. Am J Clin Nutr 2017; 105: 1351-1361

[146] Sutton EF, Beyl R, Early KS et al. Early Time-Restricted Feeding Improves Insulin Sensitivity, Blood Pressure, and Oxidative Stress Even without Weight Loss in Men with Prediabetes. Cell Metab 2018; 27: 1212-1221.e3

[147] Ravussin E, Beyl RA, Poggiogalle E et al. Early Time-Restricted Feeding Reduces Appetite and Increases Fat Oxidation But Does Not Affect Energy Expenditure in Humans. Obesity (Silver Spring) 2019; 27: $1244-1254$

[148] Mattson MP, Allison DB, Fontana L et al. Meal frequency and timing in health and disease. Proc Natl Acad Sci U S A 2014; 111: 1664716653

[149] Bo S, Fadda M, Castiglione A et al. Is the timing of caloric intake associated with variation in diet-induced thermogenesis and in the metabolic pattern? A randomized cross-over study. Int J Obes (Lond) 2015; 39: 1689-1695

[150] Henry C], Kaur B, Quek RYC. Chrononutrition in the management of diabetes. Nutr Diabetes 2020; 10: 6

[151] Wilkinson M], Manoogian ENC, Zadourian A et al. Ten-Hour Time-Restricted Eating Reduces Weight, Blood Pressure, and Atherogenic Lipids in Patients with Metabolic Syndrome. Cell Metab 2020; 31: 92-104.e5 
[152] Trepanowski JF, Kroeger CM, Barnosky A et al. Effect of Alternate-Day Fasting on Weight Loss, Weight Maintenance, and Cardioprotection Among Metabolically Healthy Obese Adults: A Randomized Clinical Trial. JAMA Intern Med 2017; 177: 930-938

[153] Sundfør TM, Svendsen M, Tonstad S. Effect of intermittent versus continuous energy restriction on weight loss, maintenance and cardiometabolic risk: A randomized 1-year trial. Nutr Metab Cardiovasc Dis 2018; 28: 698-706

[154] Schübel R, Nattenmüller J, Sookthai D et al. Effects of intermittent and continuous calorie restriction on body weight and metabolism over 50 wk: a randomized controlled trial. Am J Clin Nutr 2018; 108 : 933-945

[155] Carter S, Clifton PM, Keogh JB. Effect of Intermittent Compared With Continuous Energy Restricted Diet on Glycemic Control in Patients With Type 2 Diabetes: A Randomized Noninferiority Trial. JAMA Netw Open 2018; 1: e180756

[156] Corley BT, Carroll RW, Hall RM et al. Intermittent fasting in Type 2 diabetes mellitus and the risk of hypoglycaemia: a randomized controlled trial. Diabet Med 2018; 35: 588-594

[157] Horne BD, Grajower MM, Anderson JL. Limited Evidence for the Health Effects and Safety of Intermittent Fasting Among Patients With Type 2 Diabetes. JAMA 2020

[158] Lawrence JM, Liese AD, Liu L et al. Weight-loss practices and weightrelated issues among youth with type 1 or type 2 diabetes. Dia Care 2008; 31: 2251-2257

[159] Kahkoska AR, Watts ME, Driscoll KA et al. Understanding antagonism and synergism: A qualitative assessment of weight management in youth with Type 1 diabetes mellitus. Obes Med 2018; 9: 21-31

[160] Rosenfalck AM, Almdal T, Viggers L et al. A low-fat diet improves peripheral insulin sensitivity in patients with Type 1 diabetes. Diabet Med 2006; 23: 384-392

[161] Ranjan A, Schmidt S, Damm-Frydenberg C et al. Low-Carbohydrate Diet Impairs the Effect of Glucagon in the Treatment of InsulinInduced Mild Hypoglycemia: A Randomized Crossover Study. Diabetes Care 2017; 40: 132-135

[162] Nielsen JV, Jönsson E, Ivarsson A. A low carbohydrate diet in type 1 diabetes: clinical experience - a brief report. Ups J Med Sci 2005; 110 : 267-273

[163] Burge MR, Castillo KR, Schade DS. Meal composition is a determinant of lispro-induced hypoglycemia in IDDM. Diabetes Care 1997; 20: 152-155

[164] Ranjan A, Schmidt S, Damm-Frydenberg C et al. Short-term effects of a low carbohydrate diet on glycaemic variables and cardiovascular risk markers in patients with type 1 diabetes: A randomized open-label crossover trial. Diabetes Obes Metab 2017; 19: 1479-1484

[165] Rabasa-Lhoret R, Garon J, Langelier $\mathrm{H}$ et al. Effects of meal carbohydrate content on insulin requirements in type 1 diabetic patients treated intensively with the basal-bolus (ultralente-regular) insulin regimen. Diabetes Care 1999; 22: 667-673
[166] Anderson JW, Zeigler JA, Deakins DA et al. Metabolic effects of highcarbohydrate, high-fiber diets for insulin-dependent diabetic individuals. Am J Clin Nutr 1991; 54: 936-943

[167] McKewen MW, Rehrer NJ, Cox C et al. Glycaemic control, muscle glycogen and exercise performance in IDDM athletes on diets of varying carbohydrate content. Int J Sports Med 1999; 20: 349-353

[168] Zavitsanou S, Massa J, Deshpande S et al. The Effect of Two Types of Pasta Versus White Rice on Postprandial Blood Glucose Levels in Adults with Type 1 Diabetes: A Randomized Crossover Trial. Diabetes Technol Ther 2019; 21: 485-492

[169] Rilstone S, Reddy M, Oliver N. Glycemic Index, Extended Bolusing, and Diabetes Education in Insulin Pump Therapy (GLIDE: A Pilot Study). Diabetes Technol Ther 2019; 21: 452-455

[170] Bruttomesso D, Tessari P. A High-Fiber Diet Decreases Postabsorptive Protein Turnover but Does Not Alter Insulin Sensitivity in Men with Type 1 Diabetes Mellitus. J Nutr 2019; 149: 596-604

[171] Thinggaard $M$, Jacobsen $R$, Jeune $B$ et al. Is the relationship between $\mathrm{BMI}$ and mortality increasingly $\mathrm{U}$-shaped with advancing age? A $10 y e a r$ follow-up of persons aged 70-95 years. J Gerontol A Biol Sci Med Sci 2010; 65: 526-531

[172] Guigoz Y, Vellas BJ. Malnutrition in old age: The Mini Nutritional Assessment (MNA). Ther Umsch 1997; 54: 345-350

[173] Rubenstein LZ, Harker JO, Salvà A et al. Screening for undernutrition in geriatric practice: developing the short-form mini-nutritional assessment (MNA-SF). J Gerontol A Biol Sci Med Sci 2001; 56: M366-M372

[174] Diabetes and Nutrition Study Group of the Spanish Diabetes Association (GSEDNu) J Dibetes Complications 2006; 20: 361-366

[175] Strychar I, Cohn JS, Renier G et al. Effects of a diet higher in carbohydrate/lower in fat versus lower in carbohydrate/higher in monounsaturated fat on postmeal triglyceride concentrations and other cardiovascular risk factors in type 1 diabetes. Diabetes Care 2009; 32: 1597-1599

[176] Donaghue KC, Pena MM, Chan AK et al. Beneficial effects of increasing monounsaturated fat intake in adolescents with type 1 diabetes. Diabetes Res Clin Pract 2000; 48: 193-199

[177] Bozzetto L, Alderisio A, Giorgini M et al. Extra-virgin olive oil reduces glycemic response to a high-glycemic index meal in patients with type 1 diabetes: a randomized controlled trial. Diabetes Care 2016; 39: $518-524$

[178] Tetzschner R, Nørgaard K, Ranjan A. Effects of alcohol on plasma glucose and prevention of alcohol-induced hypoglycemia in type 1 diabetes-A systematic review with GRADE. Diabetes Metab Res Rev 2018; 34

[179] Zare Javid A, Aminzadeh M, Haghighi-Zadeh MH et al. The Effects of Synbiotic Supplementation on Glycemic Status, Lipid Profile, and Biomarkers of Oxidative Stress in Type 1 Diabetic Patients. A PlaceboControlled, Double-Blind, Randomized Clinical Trial. Diabetes Metab Syndr Obes 2020 13: 607-617 